\title{
Hematological Evaluation of Three Different Treatment Regimens in Diaphramatic Hernia in Buffaloes
}

\author{
Lokesh $^{1}$, Anup Yadav ${ }^{2 *}$, Praveen Kumar $^{3}$, Umed Singh Mehra ${ }^{2}$, Rishi Tayal ${ }^{1}$, \\ Rajendra Yadav ${ }^{4}$ and Pankaj Kumar ${ }^{5}$
}
${ }^{1}$ Department of Veterinary Surgery, ${ }^{2}$ Department of Veterinary Gynaecology and Obstetrics,
${ }^{3}$ Department of Veterinary Medicine, (LUVAS, Hisar), Haryana, India
${ }^{4}$ RVDEC, Mahendergarh (LUVAS, Hisar), Haryana, India
${ }^{5}$ Disease Investigation Laboratory, Rohtak (LUVAS, Hisar), Haryana, India

*Corresponding author

\begin{abstract}
A B S T R A C T
The present study was concluded to document the hematological evaluation of three different treatment regimens in clinical cases of diaphragmatic hernia in buffaloes which

were brought to TVCC, LUVAS Hisar during the research period. Three different NSAIDs were tried and categorized in three groups along with combination of other drugs. The investigation included haemoglobin and packed cell volume as comparative measure for the hematological analysis. No significant variation in haemoglobin and packed cell volume was noticed during the entire period of observation in all the treatment groups. Decrease in the haemoglobin and PCV concentrations in the immediate post-operative period could be due to blood loss during surgery, increased secretion of $\mathrm{ADH}$ leading to fluid retention and haemodilution also possibly because of perioperative fluid line establishment. The present study concluded that amid of various physiological differences in the buffalo suffering from diaphragmatic hernia, there are non-significance changes in the hematological parameters. After treatment with 3 different kinds of NSAID'S hematological findings were similar. So in treatment respect of D.H in buffalo hematological findings can be neglected.
\end{abstract}

Keywords

Hematological

Evaluation, Treatment

Regimens, Diaphramatic

Hernia, Buffaloes

Article Info

Accepted:

22 October 2018

Available Online:

10 November 2018

\section{Introduction}

Diaphramatic hernia is a serious digestive disorder of buffaloes, with high prevalence reported especially from North India. It is characterized by recurrent tympany, reduced milk yield, anorexia, and scanty hard black faeces. It involves a rupture in the diaphragm at the musculotendinous junction with subsequent herniation of the abdominal organs into the thoracic cavity (Singh et al., 2006). In buffaloes herniation occurs mostly through right ventro-medial part of the diaphragm, which ruptures from the musculo-tendinous junction (Singh et al., 1977; Krishnamurthy et al., 1985; Saini et al., 2000). The situation of diaphragmatic hernia is serious, as if it remains untreated causes death in $100 \%$ cases 
(Krishnamurthy et al., 1985). Treatment of diaphragmatic hernia requires surgery in two stages. The first stage involves a laparorumenotomy that enables the surgeon to assess the location and extent of herniation, retract and remove foreign bodies, if any and evacuate the rumen contents. This facilitates manoeuvres during herniorrhaphy and allows and helps to avoid regurgitation. The repair is done 24 to 48 hours after laparo-rumenotomy. During this period the animals are strictly kept off feed and water but maintained on intravenous fluid therapy. During the second stage of surgery, the repair of the diaphraghmatic defect is done under general anaesthesia along with controlled ventilation (Singh et al., 2006). There is severe pain during the time of diaphragmatic herniorrhaphy. To achieve anaesthesia and relieve the pain different analgesic drugs are used which act on peripheral and central nervous system.

General anaesthesia is a state of reversible unconsciousness produced by a process of controlled, reversible drug-induced intoxication of the central nervous system in which the patient neither perceives nor recalls noxious stimuli (Hall et al., 2001). It is not easy to select a drug or a combination of drugs for general anaesthesia especially in ruminants. The basic problem lies in the fact that there are large species variations in response to a drug. Variations are also observed even amongst the breeds within a species. Therefore, it may be dangerous to extrapolate data from one species to another for clinical application. Due to this reason, it becomes imperative to evaluate each sedative or preanaesthetic drug in each species before it can be recommended for clinical use. A variety of drugs are given to the patients that have different effects with overall aim of ensuring unconsciousness, amnesia, relaxation and analgesia. The anaesthetist selects the optimal technique for any given patient and procedure. General anaesthesia in bovines causes various complications like excessive salivation, regurgitation, tympany and cardiopulmonary depression. So a multiple drug approach (balanced anaesthesia and analgesia) is exploited to minimize and attenuate sensory, motor, sympathetic and parasympathetic reflex activities. General anaesthesia can be induced by intravenous or intramuscular injection or breathing a volatile anaesthetic agent (inhalation induction). Onset of anaesthesia is faster with intravenous (IV) injection than with inhalation taking about 1020 seconds to induce total unconsciousness. This has the advantage of avoiding the excitatory phase of anaesthesia and thus reduces complications related to induction of anaesthesia.

Glycopyrrolate has a powerful and prolonged antisialogogue activity and is about five times as potent as Atropine (Hall et al., 2001a). It is a preanaesthetic drug which stimulates cardiac reflex and blocks vagus reflex, thus prevents or inhibits cardiac inhibitory effect of Xylazine. Xylazine administration produces profuse salivation in cattle as recorded by Kumar and Singh (1979). It produced tachycardia (Khan et al., 2007a) in buffalo calves and that it when administered after Xylazine administration caused increased heart rate and mean arterial pressure (Khan et al., 2007b). Disadvantage of Glycopyrrolate to produce tachycardia and also associated to increase in oxygen demand which may result in arrhythmias or reduced cardiac contractility (Hall and Clarke, 1991). GlycopyrrolateAcepromazine-Xylazine-Thiopentone and Glycopyrrolate-Acepromazine- XylazineKetamine combination offset the bardycardiac effects of Acepromazine and Xylazine and maintained heart rate and respiratory rate within normal limits throughout the period of observation in buffaloes undergoing diaphragmatic herniorrhaphy (Ninu, 2009). Glycopyrrolate-Xylazine-Pentazocine/ 
Butorphanol- Ketamine were considered safe during diaphragmatic herniorrhaphy (Satbir, 2011).

Sedative and analgesic activity is related to CNS depression mediated by stimulation of $\alpha 2$-adrenocepters. Muscle relaxation is caused by inhibition of intraneural transmission within CNS. Xylazine is typical $\alpha_{2}-$ adrenoceptor agonist and exerts its effect accordingly. There is marked variation in susceptibility to Xylazine's effect in various species of domestic animals. In cattle administration of Xylazine $(0.5 \mathrm{mg} / \mathrm{kg}, \mathrm{IM})$ produced deep sedation with animal going into lateral recumbency (Hall and Clarke, 1969).

Atropine-butorphanol-xylazine-ketaminepropofol was found safe anaesthetic combination in buffaloes undergoing diaphragmatic herniorraphy (Pushpender, 2012). Atropine-Xyalazine-PentazocinePropofol was found better anaesthetic combination followed by Atropine-XyalazineButorphanol-Propofol and AtropineXyalazine-ketoprfen-Propofol (Kumar, 2012).

Ketamine is a dissociative anaesthetic that is used for induction/maintenance of anaesthesia in many species. It has the tendency to cause catalepsy and occasional seizers (Pageat, 1986). Pathak et al., (1982) reported the drawback of muscular rigidity with Ketamine. These demerits potentiate the necessity for the use of preanaesthetics having muscle relaxation properties along with or prior to induction and or maintenance of anaesthesia with Ketamine. Propofol was found safe intravenous anaesthetic to induce general anaesthesia in buffaloes (Ratnesh, 2010).

Although a common concern for the welfare of the animals is shared, the prevention and alleviation of pain has not always been implemented effectively. Misconceptions about the nature and significance of pain, difficulties in recognizing its severity and uncertainly as to the most appropriate therapy have all hampered progress in this area, particularly in bovines.

Nonsteroidal anti-inflamatory drugs (NSAIDs) are used extensively in veterinary practice for their analgesic, anti-inflammatory and antipyretic effects in post-operative duration.

Meloxicam is an oxicam (enolic acid) derivative. Meloxicam has preferential COX-2 activity and is also a mild inhibitor of COX-1 in platelets and kidneys (Deneuche et al., 2004). It is indicated for the management of inflammation and pain arising from acute and chronic diseases (Doig et al., 2000; Lascelles et al., 2001).

Flunixin-meglumine is a carboxylic acid nonsteroidal anti-inflammatory drug and a potent inhibitor of cyclooxygenase (Lees et al., 1987; Semrad et al., 1985). In horses, Flunixin-meglumine is indicated for alleviation of inflammation and pain associated with musculoskeletal disorders and visceral pain associated with pain.

Tolfenamic acid belongs to the fenamate group and is a potent inhibitor of cyclooxygenase enzyme. It is efficient in treatment of chronic and acute painful locomoter syndromes in the dog (Lecoindre et al., 1995).

Meloxicam, Tolfenamic acid and Flunixinmeglumine have not been tried earlier as preemptive analgesia in combination with Glycopyrrolate-Xylazine-Ketamine

anaesthesia in buffaloes undergoing diaphragmatic harniorrhaphy (D.H.). Therefore, the present study was undertaken with the following objectives:

Evaluation of Glycopyrrolate - Xylazine Meloxicam - Ketamine in buffaloes undergoing diaphragmatic herniorrhaphy. 
Evaluation of Glycopyrrolate - Xylazine Tolfenamic acid - Ketamine in buffaloes undergoing diaphragmatic herniorrhaphy.

Evaluation of Glycopyrrolate -Xylazine Flunixin-meglumine - Ketamine in buffalo undergoing diaphragmatic herniorrhaphy.

\section{Materials and Methods}

The present investigation was conducted on eighteen clinical cases of buffaloes suffering from diaphragmatic hernia presented to the Teaching Veterinary Clinical Complex, LUVAS, Hisar from August 2013 to May 2014.

Diaphragmatic hernia was confirmed by radiography and rumenotomy. In positive cases, the ruminal contents were evacuated completely by laparo-rumenotomy and thereafter diaphragmatic hernia repair was done under general anesthesia. Each animal was weighed before the diaphragmatic herniorraphy for calculating the proper dose of drugs used for general anesthesia and kept off feed and water after laparo-rumenotomy and these animals were kept strictly on fluid therapy to avoid regurgitation during operation.

The animals were divided into three groups of six animals each. Pilot trials were done to standardize the dose rates and route of administration of different drugs. Rectal temperature, heart rate (by auscultation) and respiration rate were recorded just before administration of the $\operatorname{drug}(\mathrm{s})$ along with the ambient temperature to form the base values. The blood samples were collected from jugular venipuncture before rumenotomy i.e. before administration of the drugs, before diaphragmatic herniorraphy, 15 minutes after administration of Meloxicam/Tolfenamic acid/Flunixin-meglumine, 5 minutes after administration of Ketamine, after complete recovery from the effects of the drugs and at 24 hour after recovery. Various physiological parameters were recorded at before rumenotomy, before diaphragmatic herniorrhaphy, 15 minutes after Glycopyrrolate, 10 minutes after Xylazine, and 15 minutes after Meloxicam/Tolfenamic acid/Flunixin-meglumine administration at recovery and 24 hours after diaphragmatic herniorrhaphy.

The blood samples were collected in EDTA test tubes to determine haematological parameters. After collection of blood samples, drugs were administered in accordance with the protocol. Hematological studies included estimation of hemoglobin and packed cell volume analyzed by standard methods.

\section{Statistical analysis}

The statistical analysis of data was done by one-way-analysis of variance and Duncan's multiple range test (Duncan, 1955).

\section{Results and Discussion}

The study was conducted on eighteen clinical cases of diaphragmatic hernia presented in TVCC, Hisar and these were divided into three groups of six animals each. All the parameters listed below were observed/ recorded before the animals were operated for rumenotomy and were taken as base values. The results of study are presented in tables 13.

\section{Group 1}

No significant variation in haemoglobin and packed cell volume was noticed during the entire period of observation. Post-operative hematologic data were not significantly different from baseline values in cats undergoing ovariohysterectomy with preemptive administration of meloxicam 
(Javier et al., 2008). Changes in PCV are not considered clinically important from pain management point of view as PCV is not a good indicator of post-operative pain (Mathews et al., 2001). After Xylazine administration no change in haematological values occurred in camels (Peshin et al., 1980). Contrary to this a slight reduction in haemoglobin and hematocrit occurred at maximal depth of analgesia in goats given Xylazine (Kumar and Thurmon, 1979).Decrease in the haemoglobin and PCV concentrations in the immediate post-operative period could be due to blood loss during surgery, increased secrection of ADH leading to fluid retention and haemodilution (Rosin, 1981 and Mills et al., 1992), also possibly because of perioperative fluid line establishment.

\section{Group 2}

No significant variation in haemoglobin and packed cell volume was noticed during the entire period of observation. Post-operative haematological data were not significantly different from baseline values in cats undergoing ovariohysterectomy given
Tolfenamic acid post-operatively (Javier et al., 2008). Changes in PCV are not considered clinically important from pain management point of view as PCV is not a good indicator of post-operative pain (Mathews et al., 2001). Tolfenamic acid did not cause any adverse effects on renal function, hematological variables in healthy cats (Khwanjai et al., 2012). The mean value of all hematological parameter of male and female rats of treatment group given Tolfenamic acid at 24 hours intervals for 14 days did not differ significantly from the corresponding values observed in control animals (Patel et al., 2011). After Xylazine administration no change in haematological values occurred in camels (Peshin et al., 1980). Contrary to this a slight reduction in haemoglobin and hematocrit occurred at maximal depth of analgesia in goats given Xylazine (Kumar and Thurmon, 1979). Decrease in the haemoglobin and PCV concentrations in the immediate post-operative period could be due to blood loss during surgery, increased secrection of $\mathrm{ADH}$ leading to fluid retention and haemodilution (Rosin, 1981 and Mills et al., 1992), also possibly because of perioperative fluid line establishment.

Table.1 Effects of Glycopyrrolate-Xylazine-Meloxicam-Ketamine combination on haematological parameters. Table showing arithmetic mean values obtained before and at various intervals after its administration in six buffaloes. Mean values presented with respective standard errors $( \pm)$

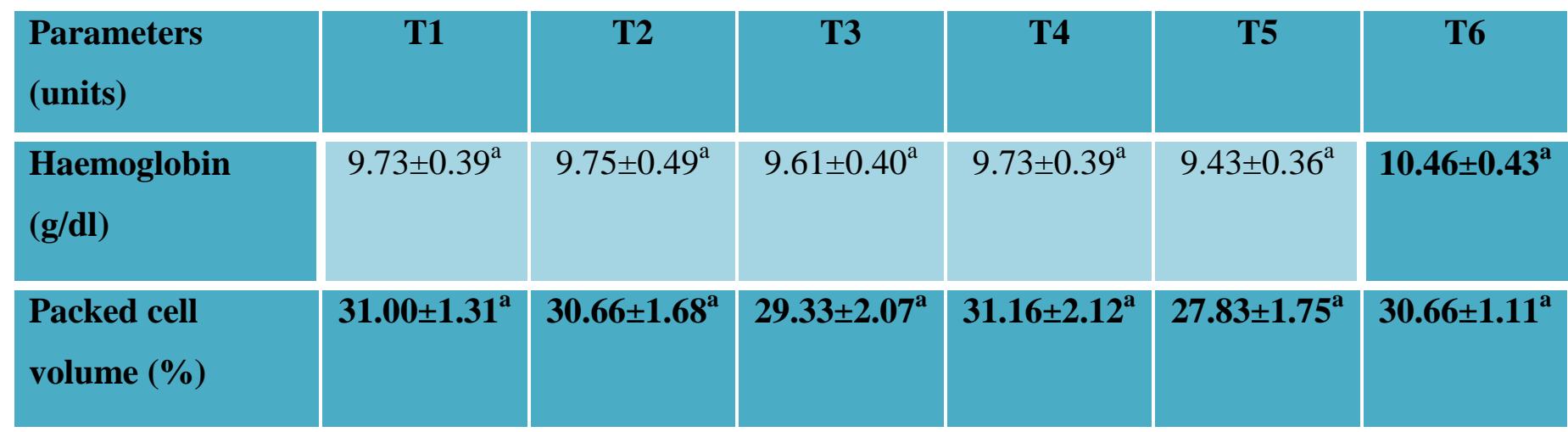

Means with same superscripts do not vary significantly ( $p>0.05)$

T1: Before Rumenotomy, T2: Before Diaphragmatic herniorrhaphy, T3: 15 minutes after Meloxicam, T4: 5 minutes after Ketamine, T5: At recovery, T6: 24 hours after Diaphragmatic herniorrhaphy. 
Table.2 Effects of Glycopyrrolate-Xylazine-Tolfenamic acid-Ketamine combination on haematological parameters. Table showing arithmetic mean values obtained before and at various intervals after its administration in six buffaloes. Mean values presented with respective standard errors $( \pm)$

\begin{tabular}{|l|c|c|c|c|c|c|}
\hline Parameters (units) & T1 & T2 & T3 & T4 & T5 & T6 \\
\hline Haemoglobin (g/dl) & $9.58 \pm 0.83^{\mathrm{a}}$ & $9.91 \pm 0.69^{\mathrm{a}}$ & $9.66 \pm 0.63^{\mathrm{a}}$ & $9.30 \pm 0.93^{\mathrm{a}}$ & $9.03 \pm 0.98^{\mathrm{a}}$ & $\mathbf{8 . 9 5} \pm \mathbf{1 . 0 1}^{\mathrm{a}}$ \\
\hline Packed cell volume (\%) & $\mathbf{2 9 . 6 6} \pm \mathbf{2 . 7 0 ^ { \mathrm { a } }}$ & $\mathbf{2 9 . 4 1} \pm \mathbf{2 . 5 6}$ & $\mathbf{2 9 . 0 0} \pm \mathbf{2 . 5 9}^{\mathrm{a}}$ & $\mathbf{2 6 . 5 0} \pm 2.75^{\mathrm{a}}$ & $\mathbf{2 6 . 0 0} \pm \mathbf{3 . 1 9}$ & $\mathbf{2 5 . 8 3} \pm \mathbf{3 . 2 4} \mathbf{4}^{\mathrm{a}}$ \\
\hline
\end{tabular}

Means with same superscripts do not vary significantly $(p>0.05)$

T1: Before Rumenotomy, T2: Before Diaphragmatic herniorrhaphy, T3: 15 minutes after Tolfenamic acid, T4: 5 minutes after Ketamine, T5: At recovery, T6: 24 hours after Diaphragmatic herniorrhaphy

Table.3 Effects of Glycopyrrolate-Xylazine-- Flunixin-meglumine-Ketamine combination on haematological parameters. Table showing arithmetic mean values obtained before and at various intervals after its administration in six buffaloes. Mean values presented with respective standard errors $( \pm)$

\begin{tabular}{|l|c|c|c|c|c|c|}
\hline Parameters (units) & T1 & T2 & T3 & T4 & T5 & T6 \\
\hline Haemoglobin (g/dl) & $10.83 \pm 0.19^{\mathrm{a}}$ & $11.06 \pm 0.52^{\mathrm{a}}$ & $11.28 \pm 1.28^{\mathrm{a}}$ & $10.53 \pm 0.48^{\mathrm{a}}$ & $10.66 \pm 0.56^{\mathrm{a}}$ & $\mathbf{1 1 . 2 3} \pm \mathbf{0 . 8 5} \mathbf{a}^{\mathrm{a}}$ \\
\hline Packed cell volume (\%) & $\mathbf{3 4 . 1 6} \pm \mathbf{0 . 9 4}$ & $\mathbf{3 6 . 0 0} \pm \mathbf{1 . 8 2}^{\mathrm{a}}$ & $\mathbf{3 6 . 5 0} \pm \mathbf{4 . 3 9 ^ { \mathrm { a } }}$ & $\mathbf{3 4 . 0 0 \pm 2 . 1 1 ^ { \mathrm { a } }}$ & $\mathbf{3 4 . 3 8} \pm \mathbf{2 . 4 4 ^ { \mathrm { a } }}$ & $\mathbf{3 5 . 5 5} \pm \mathbf{3 . 0 0} 0^{\mathrm{a}}$ \\
\hline
\end{tabular}

Means with same superscripts do not vary significantly $(p>0.05)$

T1: Before Rumenotomy, T2: Before Diaphragmatic herniorrhaphy, T3: 15 minutes after Flunixin-meglumine, T4: 5 minutes after Ketamine, T5: At recovery, T6: 24 hours after Diaphragmatic herniorrhaphy.

Following drugs were used in different combinations for repair of diaphragmatic hernia:

\begin{tabular}{|c|c|c|}
\hline Drugs & $\begin{array}{c}\text { Dose rate } \\
(\mathrm{mg} / \mathrm{kg})\end{array}$ & $\begin{array}{c}\text { Time and route of administration } \\
\text { of different drugs }\end{array}$ \\
\hline Glycopyrrolate $^{1}$ & 0.01 & Glycopyrrolate(I/M) \\
\hline Xylazine $^{2}$ & 0.04 & 20 minutes \\
\hline Meloxicam $^{3}$ & 0.5 & Xylazine(I/M) \\
\hline Tolfenamic acid $^{4}$ & 4 & 20 minutes \\
\hline Flunixin-meglumine $e^{5}$ & 2.2 & Meloxicam (I/M)/ Tolfenamic acid (I/M)/ \\
\hline Ketamine $^{6}+$ Xylazine & $1+0.04$ & Ketamine + Xylazine (I/V) \\
\hline
\end{tabular}

Analgesic repeated after 12 and 24 hours after recovery.

${ }^{1}$ Pyrolate ${ }^{R}-$ Glycopyrrolate injection U.S.P. $(0.2 \mathrm{mg} / \mathrm{ml})$, Neon Laboratories Limited, 28, Mahal Ind. Estate, M. Caves Road, Andheri [East], Mumbai - 400093, INDIA.

${ }^{2}$ Xylazine - Xylazine injection (23.32 mg/ml), Indian Immunologicals Ltd., Gollapadu, Guntur Dist- 522408 A.P.

${ }^{3}$ Aneket - Ketamine Hydrochloride Injection I.P. $50 \mathrm{mg} / \mathrm{ml}$, Neon Laboratories Limited, 28, Mahal Ind. Estate, M. Caves Road, Andheri [East], Mumbai - 400093, INDIA.

${ }^{4}$ Flunimeg ${ }^{\text {TM }}$-Flunixin-meglumine Injection I.P. 50mg/ml, Zydus AHL, Astron Tech Park, satellite cross roads Ahmedabad-380015, INDIA..

${ }^{5}$ Zobid $^{\text {TM }}{ }_{-M}$ Vet-Meloxicam injection(vet) I.P. $5 \mathrm{mg} / \mathrm{ml}$, Zydus AHL, Astron Tech Park, satellite cross roads Ahmedabad-380015, INDIA..

${ }^{6} \mathrm{Maxxtol}^{\mathrm{TM}}$-Tolfenamic injection I.P. 40mg/ml, Intas Pharmaceuticals Ltd. Matoda-382210 Ahmedabad, INDIA. 


\section{Group 3}

No significant variation in haemoglobin and packed cell volume was noticed during the entire period of observation. Changes in PCV are not considered clinically important from pain management point of view as PCV is not a good indicator of post-operative pain (Mathews et al., 2001). Satbir, 2011 reported similar findings in buffaloes undergoing diaphragmatic herniorrhaphy. There were no significant haematological differences between healthy foals treated with the recommended dosage of Flunixin-meglumine and those treated with physiological saline (Carrick et al., 1989). After Xylazine administration no change in haematological values occurred in camels (Peshin et al., 1980). Contrary to this a slight reduction in haemoglobin and hematocrit occurred at maximal depth of analgesia in goats given Xylazine (Kumar and Thurmon, 1979). Decrease in the haemoglobin and PCV concentrations in the immediate postoperative period could be due to blood loss during surgery, increased secrection of $\mathrm{ADH}$ leading to fluid retention and haemodilution (Rosin, 1981 and Mills et al., 1992), also possibly because of perioperative fluid line establishment.

The present study concluded that amid of various physiological differences in the buffalo suffering from diaphragmatic hernia, there are non-significance changes in the hematological parameters. After treatment with 3 different kinds of NSAID'S hematological findings were similar. So in treatment respect of D.H in buffalo hematological findings can be neglected.

\section{References}

Carrick, B., Mark, G., Papich; Dorothy, M., Middleton; Jonathan, M., Naylor; and Hugh, G. G. 1989. Clinical and
Pathological Effects of Flunixinmeglumine Administration to Neonatal Foals. Can J Vet Res; 53: 195-201.

Doig, P. A., Purbrick, K. A., Hare J. E. and Mckeown, D. B. 2000. Clinical efficacy and tolerance of meloxicam in dogs with chronic osteoarthritis. Can. Vet. J. 41: 296-300.

Hall, L. W. and Clarke, K. W. 1969. "Xylazine"- a new sedative for horses and cattle. Vet. Rec.85: 512-517.

Hall, L. W. and Clarke, K. W. 1991. Principles of sedation, analgesia and premedication. In: Veterinary Anaesthesia. $9^{\text {th }}$ edn. Baillire Tindal, London.pp.75-112.

Hall, L. W., Clarke, K. W. and Trim, C. M. 2001. Principle of sedation, analgesia and premedication. In: Veterinary Anaesthesia. $10^{\text {th }}$ edn. W. B. Saunders, London.

Hall, L. W., Clarke, K. W. and Trim, C. M. 2001a. Principles of sedation, analgesia and premedication. In: Veterinary Anaesthesia. $10^{\text {th }}$ edn. W. B. Saunders. London. pp. 75-112.

Javier Benito-de la-Vibora; B Duncun X Lascelles and Paloma Garcia-Fernandez 2008. Efficacy of Tolfenamic acid and meloxicam in the control of postoperative pain following ovaroihysterectomy in cat. Vet. Anaesth. Analg.35: 501-510.

Khan, I., Kumar, A; Singh, J; Peshin, P.K. and Singh, S. 2007 a. Evaluation of glycopyrrotate as an anticholinergic in buffalo calves (Bubalusbubalis). Italian J. Anim. Sci. 6(2): 1007-1010.

Khwanjai, V., Chuthatep, S., Durongphongtorn, S. and YibchokAnun, S. 2012. Evaluating the effects of 14-day oral vedaprofen and tolfenamic acid treatment on renal function, hematological and biochemical profiles in healthy cats. Vet.Pharmacol.Ther. 35(1):8-13. 
Krishnamurthy, D., Nigam, J. M., Peshin, P. K., Sharma, D. N., and Tyagi, R. P. S. 1985. Monograph on diaphragmatic hernia in bovines. Directorate of Publications, Haryana Agricultural University, Hisar, India.

Kumar, A and Singh, H. P. 1979. Ketamine and xylazine anaesthesia in bovine paediatric surgery. Indian Vet. J. 56(3): 219-222.

Kumar, A. and Thurmon, J. C. 1979. Cardiopulmonary, hemocytologic and biochemical effects of xylazine in goats. Lab. Anim. Sci. 29: 486-491.

Kumar, Sandeep 2012 Evaluation of Pentazocine lactate, Butorphanol tartrate and Ketoprofen with propofol anaesthesia in buffaloes suffering from diaphragmatic hernia. MVSc Thesis LalaLajpatRai University of Veterinary and Animal Sciences.

Lascelles, B. D. X., Henderson, A. J. and Hackett, I. J. 2001. Evaluation of the clinical efficacy of meloxicam in cats with painful locomotor disorders. $J$. Small Anim. pract. 42: 587-593.

Lecoindre, P., Thomas, E. and Richard, P. (1995). Evaluation of new therapeutic regimen of tolfenamic acid in the treatment chronic painful locomotor syndromes in dog. Proceedings of $2^{\text {nd }}$ European Congress of the Federation of European companin Animal Veterinary Associationsp. 123, Brussels 27-29 Oct 1995.

Lees, P., Ewins, C.P., Taylor, J.B.O. and Sedgwick, A.D. 1987. Serum thromboxane in the horse and its inhibition by aspirin, phenylbutazone and flunixin. Br. Vet. J., 143: 462-476.

Mathews, K. A. 2001 Perioperative use of non-steroidal anti-inflamatory analgesics. Proceedings World Small Animal Vet. Assoc. Congress. (WSAVA-2001).
Mills, D. L., Hauptman, J. G. and Richter, M. 1992. Preoperative and postoperative hemostatic profiles of dogs undergoing ovariohysterectomy. Cornell Vet. 82: 465-470.

Ninu, A. R. 2009. Evaluation of anaesthetic protocols in diaphragmatic herniorrhaphy in buffaloes (Bubalus bubalis). MVSc. Thesis. CCS Haryana Agricultural University. Hisar.

Patel, S. D., Sadariya, K. A., Gothi, A. K., Patel, U. D., Jain, M.R., Bhavsar S. K. and Thaker, A. M. 2011. Safety of tolfenamic acid following repeated intramuscular administration in wistar rats. Pharma Sci. Monitor Int. J. Pharm. Sci., 2: 79-85.

Peshin, P. K., Nigam, J. M., Singh, S.C. and Robinson, ---- B. A. 1980. Evaluation of xylazine in camels. J. Am. Vet. Med. 177(9): 875-878.

Pushpender 2012 Evaluation of XylazineKetamine-Propofol anaesthesia in buffaloes undergoing diaphragmatic herniorrhaphy. MVSc Thesis LalaLajpatRai University of Veterinary and Animal Sciences

Ratnesh, 2010. Evaluation of Propofol as intavenous anaesthetic to induce general anaaesthesia in buffalo calves. $M V S c$ Thesis, CCS Haryana Agricultural University, Hisar.

Rosin, E. 1981. The systemic response to injury. In: Pathphysiology in small animal surgery. Bojarb M.J. (Ed.), Lea and Febiger, Philadelphia, pp: 3-11.

Saini, N. S., Sobti, V. K., Mirakhur, K. K., Singh, S. S., Singh, K. I., Bansal, P. S., Singh, P. S. and Bhatia, R. 2000. Retrospective evaluation of 80 nonsurviving buffaloes with diaphragmatic hernia. Vet. Rec. 147: 275-276.

Satbir 2011. Comparative evaluation of Pentozocine lactate, Butorphanol tartrate and Dipyrone in buffaloes undergoing diaphragmatic 
herniorrhaphy. MVSc Thesis LalaLajpatRai University of Veterinary and Animal Sciences.

Semrad, S. D., Hardee, G. E., Hardee, M. M. 1985. Flunixin-meglumine given in small doses: pharmacokinetics and prostaglandin inhibition in healthy horses. Am. J. Vet. Res., 46: 2474-2479. Singh, S., Kumar, A., Singh, J., Singh, S. and Peshin, P. K. 2006. Haemodynamic effects of atropine-diazepamthiopentone anaesthesia in buffalo calves. Indian J. Vet. Res. 15(1): 22-30.

\section{How to cite this article:}

Lokesh, Anup Yadav, Praveen Kumar, Umed Singh Mehra, Rishi Tayal, Rajendra Yadav and Pankaj Kumar. 2018. Hematological Evaluation of Three Different Treatment Regimens in Diaphramatic Hernia in Buffaloes. Int.J.Curr.Microbiol.App.Sci. 7(11): 2970-2978.

doi: https://doi.org/10.20546/ijcmas.2018.711.340 